\title{
Two Kinds of Digital Video Watermarking Algorithm Implement and Compare
}

\author{
Xiao Luo ${ }^{1}$, Haifeng $\mathrm{Shi}^{1}$ and $\mathrm{De} \mathrm{Li}^{1 *}$ \\ ${ }^{1}$ Department of Computer Science, Yanbian University, Yanji, China \\ [e-mail: lxazy123@163.com,shi.haif@163.com,leader1223@ybu.edu.cn] \\ *Corresponding author: De Li (leader1223@ybu.edu.cn)
}



With the rapid development of multimedia technology, large dmounts of digital information turn into easy storage and transmission, at the same time, copyright protection and authentication become a problem to be solved Digital Vidé is composed of a series of continuous static images, because of its own chardeteristic, general image watermarking technology is difficult to direct extension to the wideo signal. In this paper, by using DCT transform and DWT transform, combined with the mean quantization algorithm, using three dimensional frequency domain transformation, introduces two kinds of transformation to the $3 D$ video watermarking technology, utilize the quantitative test method to prove the existence of the watermark, and through the affacked experiments to verify the low complexity and well imperceptibility and robustness abdut the video watermarking algorithm.

Keywords: Digital Video, 3D-DCT transform, 3D-DWT transform, Quantization detection, Robustness

\section{Introduction}

With the apid development of computer network technology and digitization of information media, the transmission and circulation of multimedia information become very convenient, we can storage, and transmission the digital video very convenient, but the subsequent copyright piracy behavior makes the copyright protection of digital video is more and more important video watermarking technology is an effective means of copyright protection, get the attention of the people increasingly, it also has become the hot topics in the study of current digital watermarking [1-3]. Video watermarking can be understood as a carrier for digital video of the subjective and the objective time redundancy and redundancy in the information space to add information, it does not affect the quality of video and also can achieve the purpose of copyright protection and content integrity test for digital watermarking technology. With the continuous development of video technology, has produced some quite mature video watermarking technology but with the emergence of a variety of attack forms and the core technology of some video encoding and decoding be made public, coupled with the continuous in-depth study of the human visual system, makes the watermark embedding scheme is becoming more and more complicated and at the same time in order to realize the integrity of the watermark detection certification, the strategy of watermark embedding and the modification technology had the great synchronous development. The requirements of video watermarking algorithm for watermarking products quality is that cannot cause big damage and the algorithm itself also requires has good invisibility and robustness so the robustness and resistance to attack is the most basic conditions of digital video watermarking scheme to protect the copyright . 
This thesis focuses on digital video, under the three dimensional frequency domain do a study using the combination of DWT and DCT [4] transform with mean quantization algorithm [5], on the basis of the realization algorithm further proceed comparison experiment. First introduce some knowledge related to digital video watermarking technology; Secondly introduces the application of frequency domain transformation and quantitative techniques as well as related algorithm implementation; In the end has carried on the contrast experiments ${ }^{[6]}$ to determine the performance of quantitative algorithm in the three dimensional frequency domain by this paper, through compared the experimental data about two kinds of algorithms to determine a kind of algorithm has better security, robustness and easy to implement.

\section{Related Work}

\subsection{Three-Dimensional Discrete Wavelet Transform}

The two-dimensional multi-resolution decomposition theoly has been widely used in image processing field and in order to realize the mati-resolution decomaposition of dynamic image (video), it is necessary to extended the two-dimensional wavelet transform algorithm is to three-dimensional. Figure 1 (a) is dynamic image signal a layer of wavelet decomposition of the three dimensional subbands and $\mathrm{x}$ direction representative the frame adept direction and $y$ direction representative the frame columndirection and $z$ direction is timeline direction. A layer of resolution based on three dimensjonal waveret transform decomposition process as shown in Figure 1 (b). In the figure 1 express the low frequency components that image sequence after low frequency filtering and $\mathrm{H}$ express the high frequency components that image sequence after high frequency filtering Similar to two-dimensional wavelet transform, image sequence after three-dimensional transform is broken down into a lower resolution close to three dimensionalsubband and a series of high frequency three dimensional subband representative of different resolution detall image sequence.

Assume that video sequences on the space of two dimensional to marked with $\mathrm{x}$ and $\mathrm{y}$, time dimension by z. Three dimensional discrete wavelet transform in the $\mathrm{x}, \mathrm{y}$, and $\mathrm{z}$ respectively to one dimensional integer wavelet transform. In the figure L express the low frequency components that image sequence after low frequency filtering and $\mathrm{H}$ express the high frequency components [7] that image sequence after high frequency filtering. Video image sequence after three dimensional transform is decomposed into approximation under a low resolution called low frequency three dimensional subband or LLL and a series of high frequency three dimensional subband representative of different resolution detail image sequence named LLH to HHH. Due to the human eye with the different frequencies the sensitivity also different and the main energy of video image signal is concentrated in the low frequency region [8], so we can repeat for the multistage integer wavelet decomposition to this part. Thus we draw the three dimensional discrete wavelet transform main principle:

First of all, we will be continuous in the video of the frame is divided into a group; Second, for each set of every frame image in the two dimensional discrete wavelet transform; Finally, choose the coefficient CA after two dimensional discrete wavelet transform in the time axis make up a one dimensional vector and to this one dimensional vector proceed one dimensional discrete wavelet transform again and will get the CA coefficient as well as a number of CD coefficients and this process is known as "three dimensional discrete wavelet transform". 


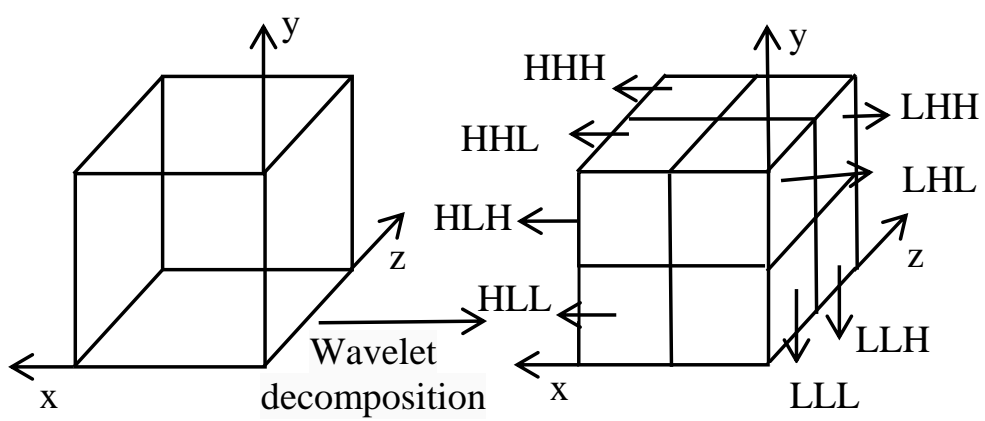

(a) The original dynamic image (b) A layer of three dimensional wavelet decomposition

Figure 1. A Layer of Wavelet Decomposition of the Three Subband

Three dimensional discrete wavelet transform process is shown in Figure 2:
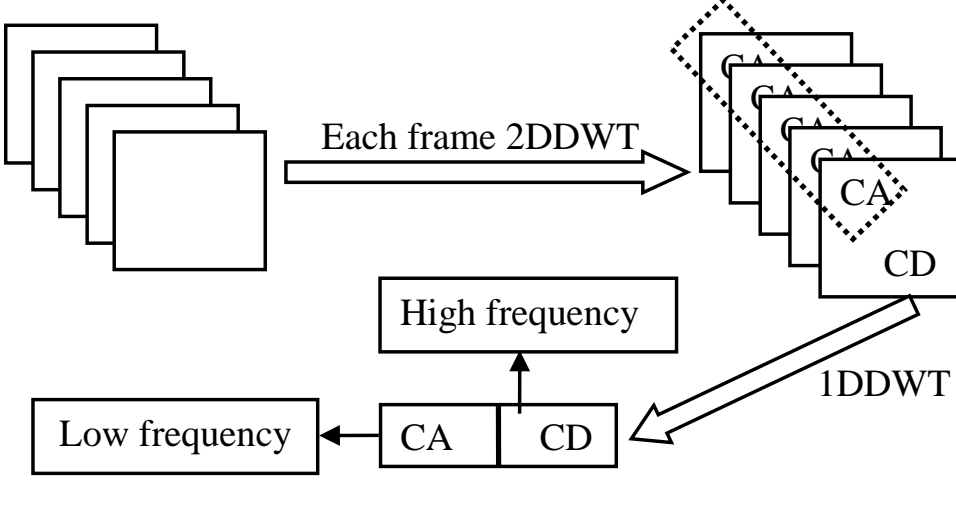

High frequency



1DDWT

Figure 2. The Basic Principle Diagram of Three Dimensional Discrete Wavelet 8 Transforms

\subsection{Three-Dimensionar Discrete Cosine Transform}

Discrete Cosine Transform referred to as DCT. Any continuous fourier transform of real symmetric function only contains cosine item so the cosine transform and fourier transform has clear physlcal meaning, discrete cosine transform to avoid the complex computation of fourien transform and it based on the orthogonal transformation of real numbers.

For the spatial domain signal sampling by discrete cosine transform and then transform them into an equivalent frequency domain.

MXN two-dimensional discrete cosine transform are defined as shown in type (1):

$$
\mathrm{F}(\mathrm{u}, \mathrm{v})=\frac{2}{\sqrt{\mathrm{MN}}} c(u) c(v) \sum_{0}^{M-1} \sum_{0}^{N-1} \mathrm{f}(x, y) \cos \frac{(2 x+1) u \pi}{2 M} \cos \frac{(2 y+1) v \pi}{2 N}
$$

The $\mathrm{x}, \mathrm{y}$ is space coordinates of sampling domain and $\mathrm{u}, \mathrm{v}$ is the coordinate of transform domain, $\mathrm{c}(\mathrm{u}), \mathrm{c}(\mathrm{v})=\left\{\frac{1}{\sqrt{2}}, i f u, v=0\right.$ inverse discrete cosine transform (IDCT) are defined as shown in type (2): 1 , ifelse 


$$
\mathrm{f}(\mathrm{x}, \mathrm{y})=\frac{2}{\mathrm{~N}} \sum_{0}^{\mathrm{M}-1} \sum_{0}^{\mathrm{N}-1} c(u) c(v) F(u, v) \cos \frac{(2 x+1) u \pi}{2 \mathrm{M}} \cos \frac{(2 y+1) v \pi}{2 N}
$$

Video image can be seen as a three-dimensional images group (the time as the third dimension). Put the image group with three dimensional discrete cosine transform in frequency domain space [9].

Three-dimensional image block $\{\mathrm{f}(\mathrm{x}, \mathrm{y}, \mathrm{z})\}$ is a matrix with $\mathrm{M}$ rows and $\mathrm{N}$ columns and $\mathrm{P}$ width, discrete cosine transform as shown in type (3):

$$
\left.\mathrm{f}(\mathrm{u}, \quad \mathrm{v}, \mathrm{w})=\sqrt{\frac{8}{\mathrm{MNP}}} c(u) c(v) c(w) \sum_{0}^{\mathrm{M}-1} \sum_{0}^{\mathrm{N}-1} \sum_{0}^{P-1} f(x, y, z) \cos \frac{(2 x+1) u \pi}{2 M} \cos \frac{(2 y+1) v \pi}{2 N} \cos \frac{(2 z+1) w \pi}{2 P}\right)
$$

Three dimensional discrete cosine inverse transform (3D-IDCT) is definied as shown in type (4) :

$$
\mathrm{f}(\mathrm{x}, \mathrm{y}, \mathrm{z})=\sqrt{\frac{8}{\mathrm{MNP}}} c(u) c(v) c(w) \sum_{0}^{\mathrm{M}-1} \sum_{0}^{\mathrm{N}-1} \sum_{0}^{P-1} f(u, v, w) \cos \frac{(2 x+1) u \pi}{2 M} \cos \frac{(2 y+1) v \pi}{2 N} \cos \frac{(2 z+1) w \pi}{2 P}
$$

From the above mentioned we can summarize the main principle of three dimensional discrete cosine transform is as follows:

First of all, we will be continuous in the video of the frame is divided into a group; Second, for every frame image in each group is divided into equal size of the block and proceed two dimensional discrete cosine transform; Finaly, choose the same set of DC coefficient of each frame in the same position to constiute the one-dimensional vector in the time axis and to this one dimensional vector proceed one dimensiongl discrete cosine transform again and will get the DC coefficient as well as a number of AC coefficients and this process is known as “ three dimensional discrete cosine transform, [10].

Three dimensional discrete cosine trânsform process as shown in Figure 3:

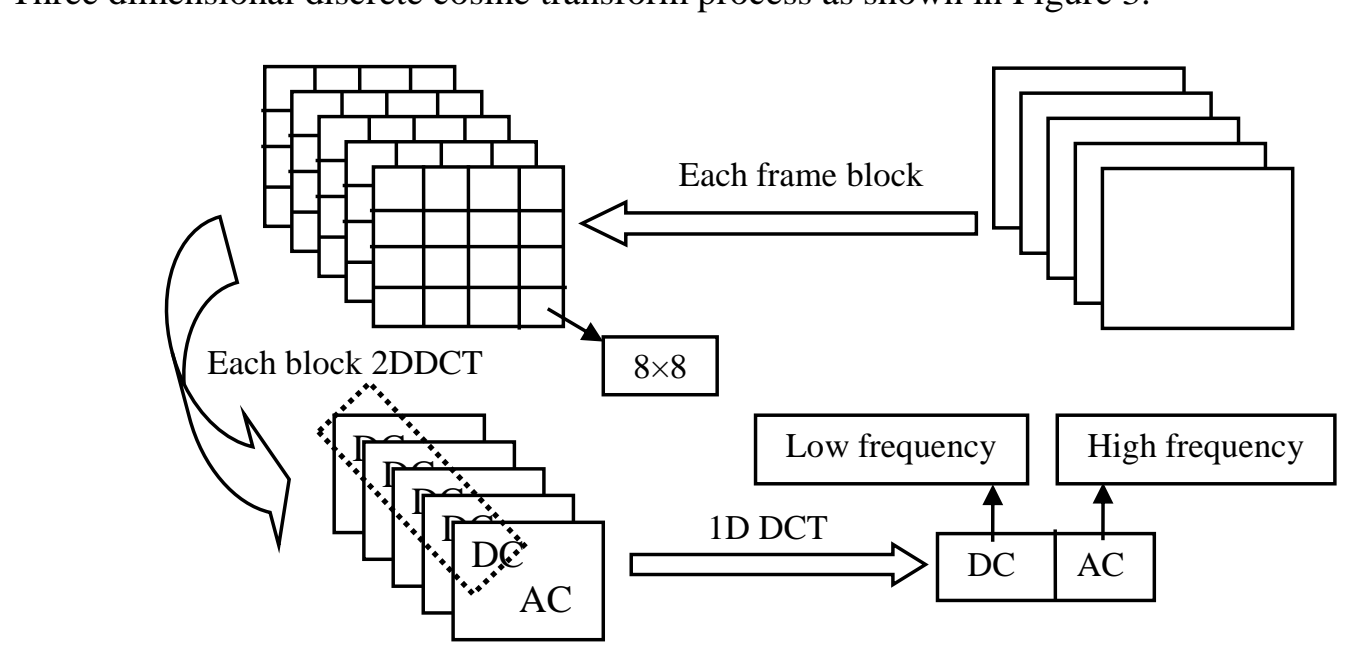

\section{Figure 3. The Basic Principle Diagram of Three Dimensional Discrete Cosine Transform}

A simplified watermarking system based on discrete cosine transform after the forward discrete cosine transform and then to reverse discrete cosine transform to gain the image that contains the watermark. The watermark extraction is carried out after forward discrete cosine transform. Movement image sequence of every frame can be regarded as a static image to three dimensional discrete cosine transform also can be regarded as each frame for first two 
dimensional discrete cosine transform and then one-dimensional discrete cosine transform in interframe direction. After two-dimensional discrete cosine transform the energy of every frame mostly focus on direct current and low frequency, which concentrated in the upper left of matrix secondary diagonal after transform, lower right after quantify vast majority is 0 [11]. Type of discrete cosine transform algorithm, the key problem is that it ignores the data loss resulted from the algorithm implementation. When no any disturbance to discrete cosine transform coefficient proceed the discrete cosine transform and then do discrete cosine inverse transform its value will fall near the each point values and error is very small just do a little processing can be reverted to the original data.

\subsection{Mean Quantization}

Set a collection composed of $\mathrm{K}$ wavelet coefficient is $\left\{x_{0}, \ldots, x_{k-1}\right\}$ and the mean is $\bar{x}=\frac{1}{K} \sum_{i=0}^{K-1} X i$, assume that the mean $\bar{x}$ using quantitative method to embed a watermark bits $\omega_{i} \in\{0,1\}$ caused by the error is $\triangle$, namely afte empedded a watermark bits $\omega_{i}$ the mean of $\mathrm{K}$ signal is $\bar{x}=\bar{x}+\triangle$ therefore $\mathrm{K}$ wavelet coefficients in the collection of all the individual coefficients also need to modify accordingly also shown ${ }^{2} x_{i}{ }^{\prime}=x_{i}+\triangle, \mathrm{i}=0, \ldots, \mathrm{K}-1$, among them $x_{i}{ }^{\prime}$ refers to a be modified coefficient values. By type (1) we can know that when embed a watermark bits in the K opeffigient of the mean $\bar{x}$ the embedded error $\triangle$ at the same time added to each component of $K$ signals namely each component $x_{i}$ has also had a $\triangle$ embedded error.

Assume that the signal of embedded watermark in the process of transmission by a certain attacks and for each signal the error is $\delta_{i}$ which is after attacks some wavelet coefficients in $\mathrm{K}$ wavelet coefficient is $X_{i}=x_{i}+\delta_{i}, \mathrm{i}=0, \ldots, \mathrm{K}-1$, after attack the mean of $\mathrm{K}$ wavelet coefficient is $\bar{X}_{\mathrm{i}}=\frac{1}{K} \sum_{i=0}^{K-1} X_{i} \gamma_{X^{\prime}}+\delta$.

Assume $\delta_{i} \sim \mathrm{N}\left(\mathrm{O}, \sigma^{2}\right)$ that is $\delta_{i}$ to obey the mean and value is 0and the variance is $\sigma^{2}$ of gaussian distribution, on the basis of probability and statistics knowledge, $\mathrm{K}$ random variables that obey gaussian distribution its average still obey gaussian distribution but the variance reduced Rtimes namely $\bar{\delta} \sim \mathrm{N}\left(\mathrm{O}, \frac{\delta^{2}}{\mathrm{~K}}\right)$.

As a esult, the mean quantization compared with single factor quantitative methods [5]can decrease the variance of coefficient change quantity and improve the robustness of the algorithm. Mean quantization method of watermark error probability is less than a single coefficient quantization caused by errors probability. Obviously, when the number of wavelet coefficients increases the watermark error probability will reduce [12]. So choose to embed a watermark bits appropriate number of wavelet coefficient can control the balance between the robustness of watermarking system and the watermark capacity.

\section{The Video Watermarking Algorithm Design}

This paper use the video format for YUV video clips to experiment, because the digital video is composed of a series of continuous static image so we should retrieve each static 
images from the video and in the form of processing of two-dimensional image to process it and then in the timeline have the second time, so completes the three-dimensional processing of for digital video. In the process of static image using quantitative algorithm proceed binary image watermark embedding.

\subsection{Three-Dimensional Discrete Wavelet Transform}

\subsubsection{Watermark Embed}

The embed watermark signal in this algorithm is not a traditional sequence code or bitstream but a binary image will be treated as watermark to processing and hidden, which makes the watermarking information contains more rich and more intuitive. Based on the three dimensional discrete wavelet transform watermarking embedding algorithm is déscribed as follows:

Step 1. Select the original video I frame and group accord to the $\mathrm{N}$ size.

Step 2. For each set of every frame proceed the two-dimensional discrete wavelet transform;

Step 3. Each set of every frame the CA coefficient restructuring into one-dimensional vector in the time axis;

Step 4. For the one dimensional vector with one-dimenstonal discrete wavelet transform and get the CA coefficient;

Step 5. Make the watermark to quantiô and embed in the each CA coefficient;

Step 6. Reconstruction the video sequences and save it [13].

Get the video file that containing the watermark

The watermark embedding proeess is showeit in Figure 4.

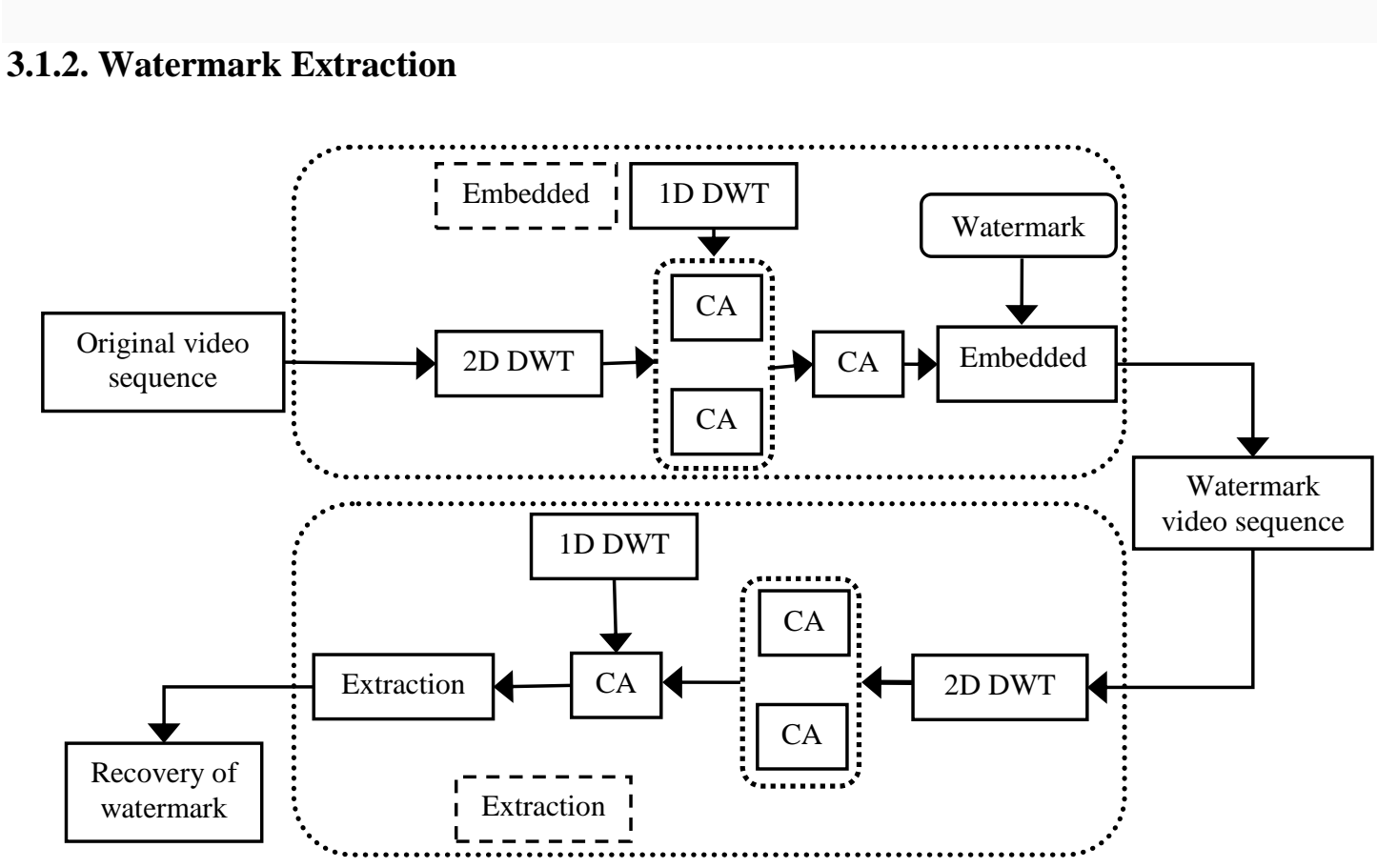

Figure 4. 3D-DWT for Video Watermarking Algorithm Description

The extraction of the watermark algorithm is the inverse process of embedded algorithm. Step 1. Select the watermark video I frame and group accord to the N size; 
Step 2. For each set of every frame proceed the two-dimensional discrete wavelet transform;

Step 3. Each set of every frame the CA coefficient restructuring into one-dimensional vector in the time axis;

Step 4. For the one dimensional vector with one-dimensional discrete wavelet transform and get the CA coefficient;

Step 5. According to the quantization algorithm get the embedding watermark.

The watermark extracting process is shown in Figure 4.

\subsection{Three-Dimensional Discrete Cosine Transform}

\subsubsection{Watermark Embed}

This algorithm to embed the watermark signal is the same as a binary image, in order to be comparative and improve the robustness of the watermark make the watemarking signal embedded into the low frequency part of discrete cosine tran form coefficients. Based on three dimensional discrete cosine transform watermarking embedding algorithm is described as follows:

Step 1. Select the original video I frame and group accord to the $\mathrm{N}$ size;

Step 2. For each set of every frame proceed the two-dimensional discrete cosine transform;

Step 3. Each set of every frame the DC coefficient restructuring into one-dimensional vector in the time axis;

Step 4. For the one dimensional vector with ore-dimensional discrete cosine transform and get the DC coefficient;

Step 5. Make the watermark to quantify andembed in the each DC coefficient;

Step 6. Reconstruction the yideo sequences and save it.

Get the video file that containing the watermark.

The watermark embedding process is shown in Figure 5.

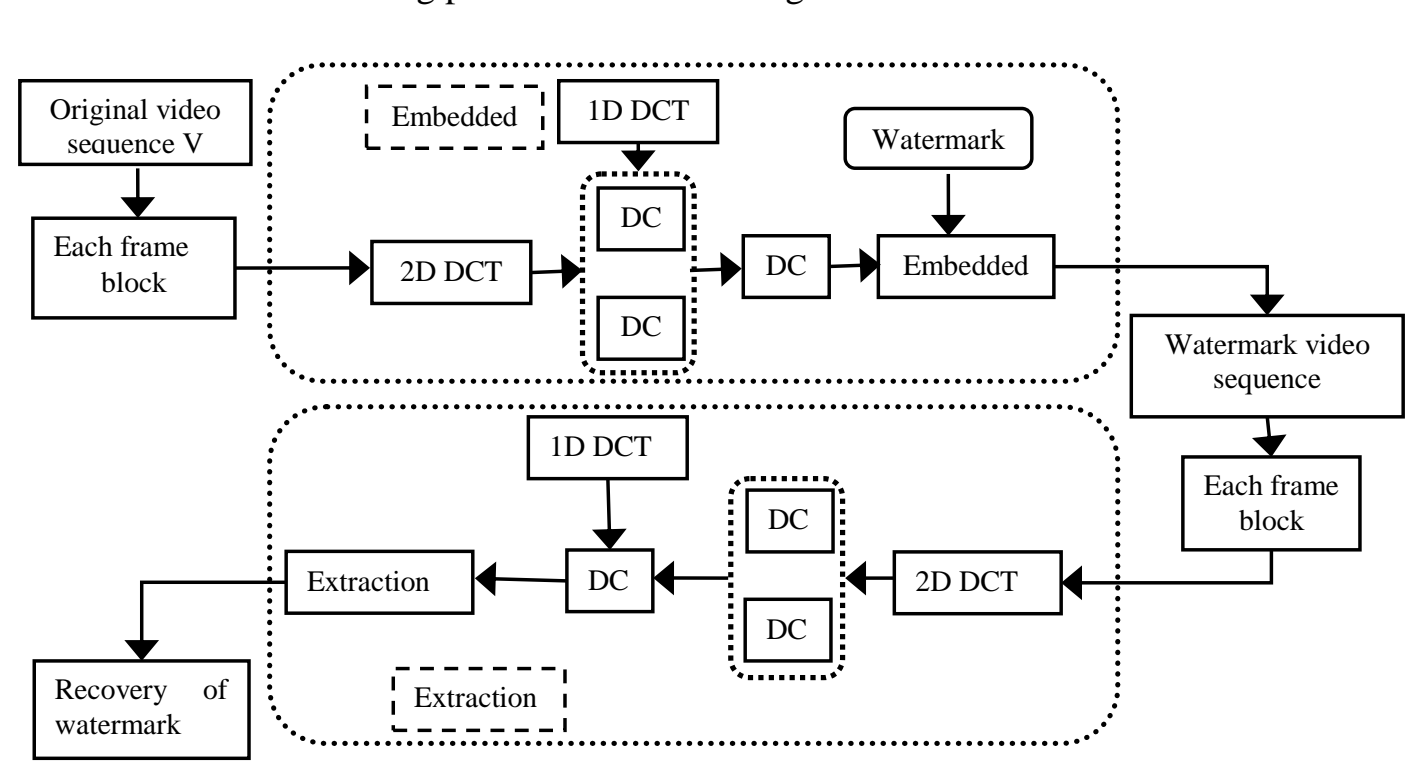

Figure 5. 3D-DCT for Video Watermarking Algorithm Description

\subsubsection{Watermark Extraction}

The extraction of the watermark algorithm is the inverse process of embedded algorithm : 
Step 1. Select the watermark video I frame and group accord to the $\mathrm{N}$ size;

Step 2. For each set of every frame proceed the two-dimensional discrete wavelet transform;

Step 3. Each set of every frame the CA coefficient restructuring into one-dimensional vector in the time axis;

Step 4. For the one dimensional vector with one-dimensional discrete wavelet transform and get the CA coefficient;

Step 5. According to the quantization algorithm get the embedding watermark.

The watermark extracting process is shown in figure 5.

\section{The Experiment Results and Analysis}

In order to verify the performance of the two kinds of video watermarking algorithm, we had a large number of experiments under MatlabR2011b. Mainly attack includes the gauss noise and salt and pepper noise as well as exchange or loss of frame.

In order to ensure comparability of the test results these two kinds of video watermarking algorithm are composed of 100 frames by the size of $176 \times 144$ uncompressed video file to experiment and decide to extract the 20th frame as a reference as shown in Figure 6. Watermark image is $18 \times 22$ size of binary image as shown in Figure 7.

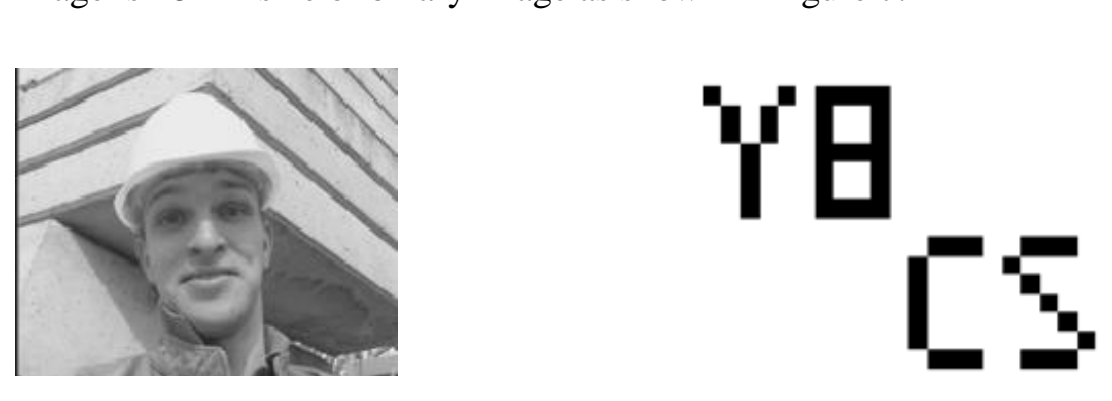

Figure 6, Video file 20th frame

Figure 7. Binary watermark image

Because robustness and no perceptual is very important factor in measure the watermark performance, so in the experiment we used the peak signal-to-noise ratio (PSNR) to measure the no perceptual of watermark, if PSNR is greater than 40dB, we can think the algorithm with a good no perceptual. The calculation of PSNR as shown in formula (5) among it the $p$ and represent the original image and the $p$ ' image represent the image after the transformation, $\mathrm{M}$ and $\mathrm{N}$ show image size.

$$
\text { PSNR }=\operatorname{lol}_{10}\left(\frac{255^{2}}{\frac{1}{M N} \sum_{x, y}\left(P(i, j)-P^{\prime}(i, j)\right)^{2}}\right)
$$

Using normalized correlation (NC) and bit error rate (BER) to measure the robustness of the watermark [14]. NC calculation as shown in formula (6), w represent the original watermark and $\mathrm{w}$ 'represent the extracted watermark, $\mathrm{M}$ and $\mathrm{N}$ show the size of the watermark.

$$
N C\left(w, w^{\prime}\right)=\frac{\sum_{i=0}^{M-1} \sum_{j=0}^{N-1} w(i, j) \cdot w^{\prime}(i, j)}{\sqrt{\sum_{i=0}^{M-1} \sum_{j=0}^{N-1} w^{2}(i, j)} \sqrt{\sum_{i=0}^{M-1 N-1} \sum_{j=0}^{N-1} w^{\prime 2}(i, j)}}
$$


The calculating of BER as shown in formula (7), the letters meaning same as NC formula.

$$
B E R=\frac{\sum_{i=1}^{M \times N} w_{i} \oplus w_{i}^{\prime}}{M \times N} \times 100 \%
$$

After using the two kinds of algorithms embed watermark, the average PSNR values of containing watermark video files were higher than $40 \mathrm{~dB}$, in the absence of any attacks can be fully extracted the watermark and $\mathrm{NC}$ equal to 1 and BER equal to 0, in the case of PSNR approximately equal to proceed the attack experiment.

In this paper the experimental condition is video watermark carrier for the same/videofile and watermark image also for the same binary image, under the condition of using the two kinds of video watermarking algorithm to embedding watermarking. In order to ensure the strong comparative of attack result, by adjusting the watermark embedding strength makes the average PSNR of video file containing the watermark about 41.17dB. We choose the 20th frame image as a reference to contrast and distinguish between two kinds of video watermarking algorithm as shown in the following Table 1 , under the condition of without attack for video file to read the containing watermark image video frames and extract the binary watermark image, shows two watermarking algorithm could extracted watermark image in good condition.

To verify two kinds of video watermarking algorithm robustness against noise attack, chose the gauss-noise and salt and pepper noise attacks on video experiment, the experimental results respectively in the following Table 2, Table 3 as shown in table.

As can be seen from Table 2, wheh applying a Yarger degree of gauss-noise attack and even affect the sensory effects of the ordginal video frame, we can still get a recognizable image watermark to prove the ownership of the digital works, which have the effect of copyright protection.

Table 1. Recovered Results of None Attacked

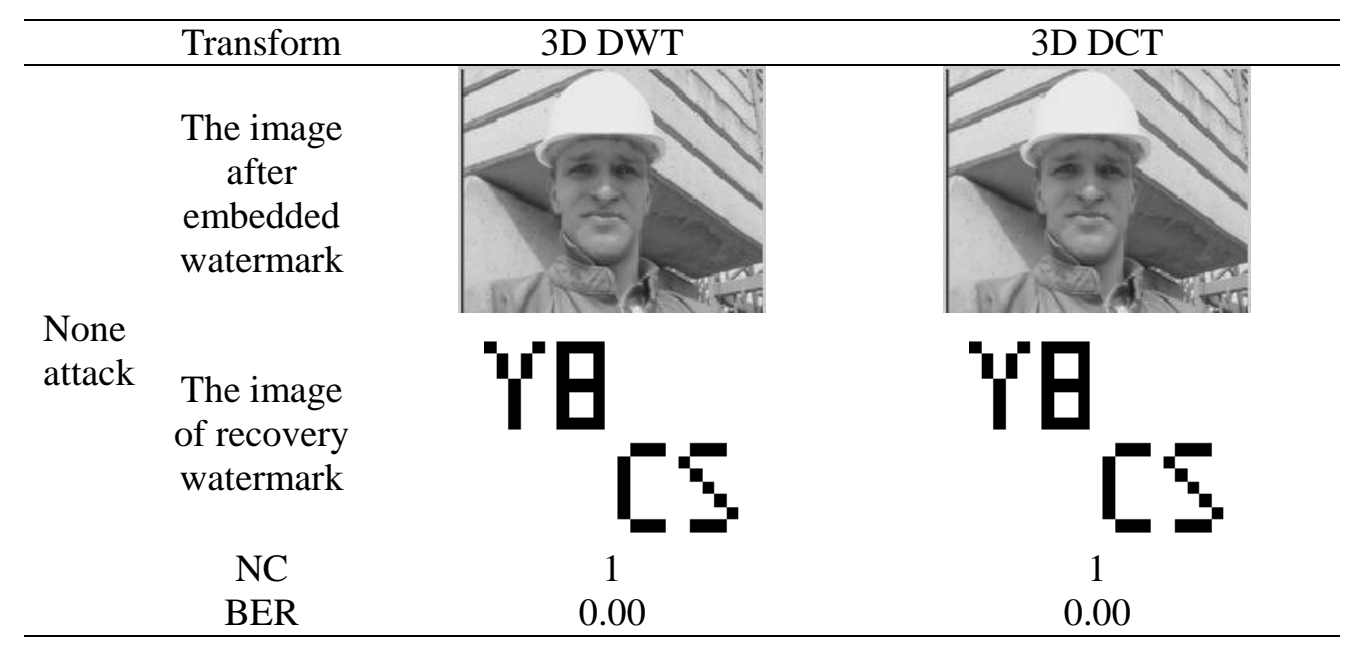


Table 2. Recovered Results of Gaussian Noise Attacked

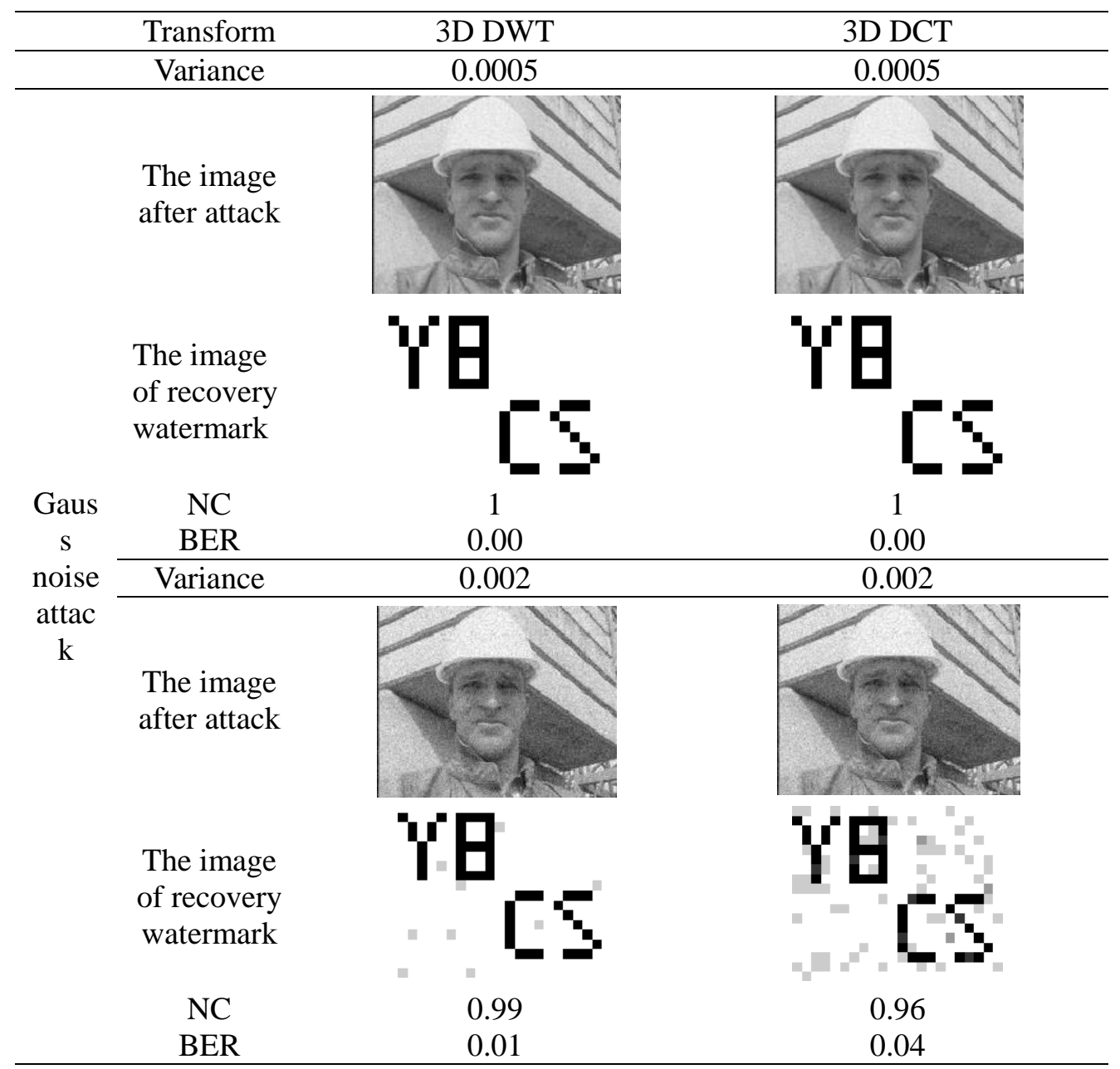

Table 3.Recovered Results of Pepper Noise Attacked

\begin{tabular}{|c|c|c|c|}
\hline & $T_{\mathrm{m}}^{\text {Transfor }}$ & 3D DWT & 3D DCT \\
\hline & Density & 0.002 & 0.002 \\
\hline $\begin{array}{l}\text { Salt } \\
\text { and } \\
\text { pepper }\end{array}$ & $\begin{array}{l}\text { image } \\
\text { after } \\
\text { attack }\end{array}$ & & \\
\hline & $\begin{array}{c}\text { image of } \\
\text { recovery } \\
\text { watermar } \\
\text { k }\end{array}$ & & \\
\hline & $\mathrm{NC}$ & 0.989 & 0.990 \\
\hline
\end{tabular}




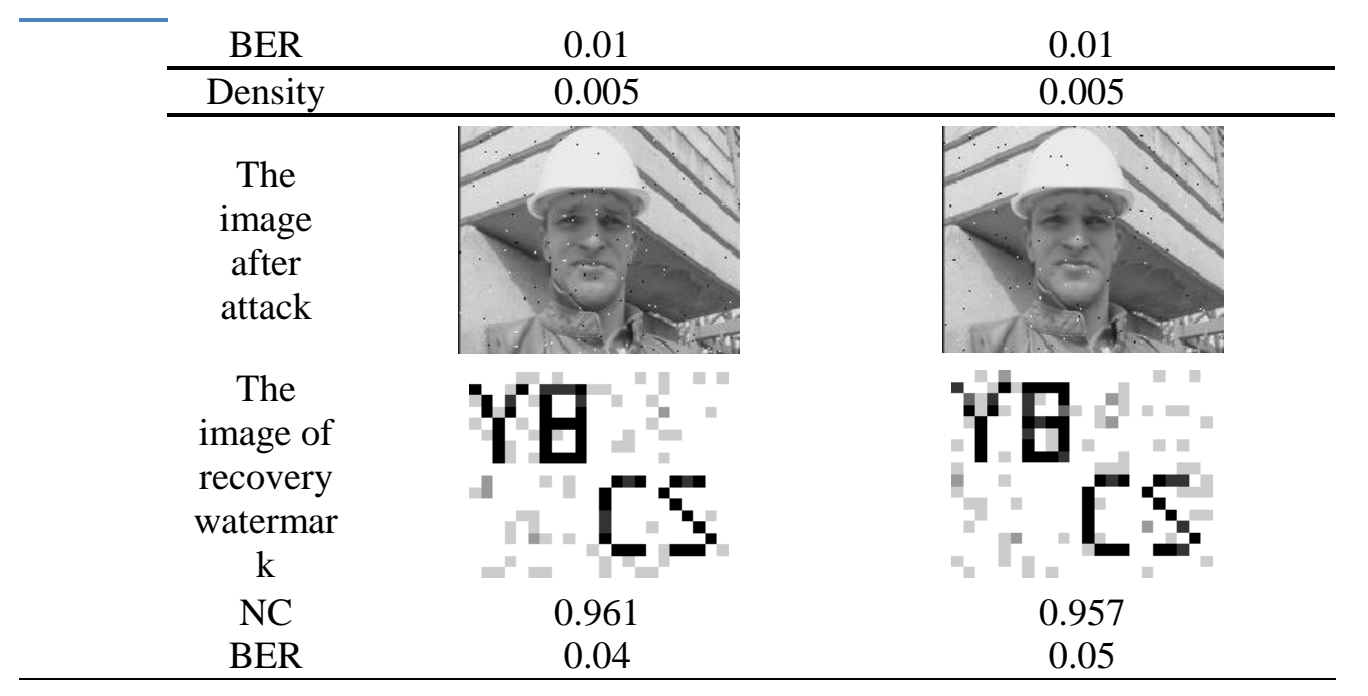

As can be seen from Table 3, when applying a larger degree of salt and pepper noise attack and even affect the sensory effects of the origina image, we can still get a recognizable watermark image. Compared with gauss-noise attack two kinds of video watermarking algorithm for salt and pepper noise resistance@ility are weak.

In order to verify the algorithm robustness in frame exehange attack, separately to two video watermarking algorithm for frame exchange experiment and results as shown in Table 4.

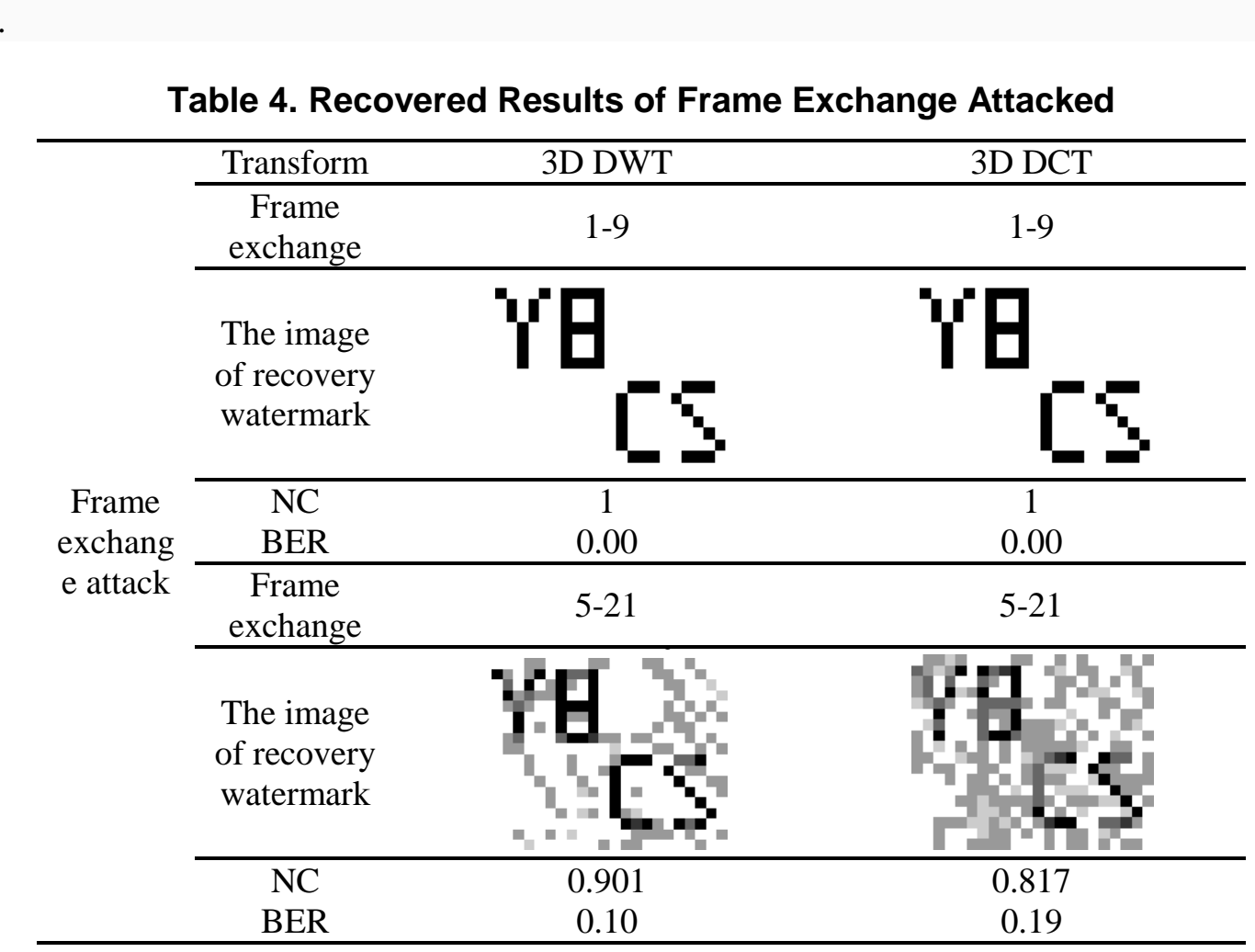

As you can see from the Table 4, no matter exchange for same group video frames exchange or exchange for different groups video frames can get a relatively clear watermark image. When exchange the same group video frames, the extraction effect is not big but if it is in a different group the impact is bigger. 
In order to verify the algorithm robustness in resisting frame loss attack, carried out the frame loss experiment and the results are shown in table 5.

\section{Table 5. Recovered Results of Frame Delete Attacked}

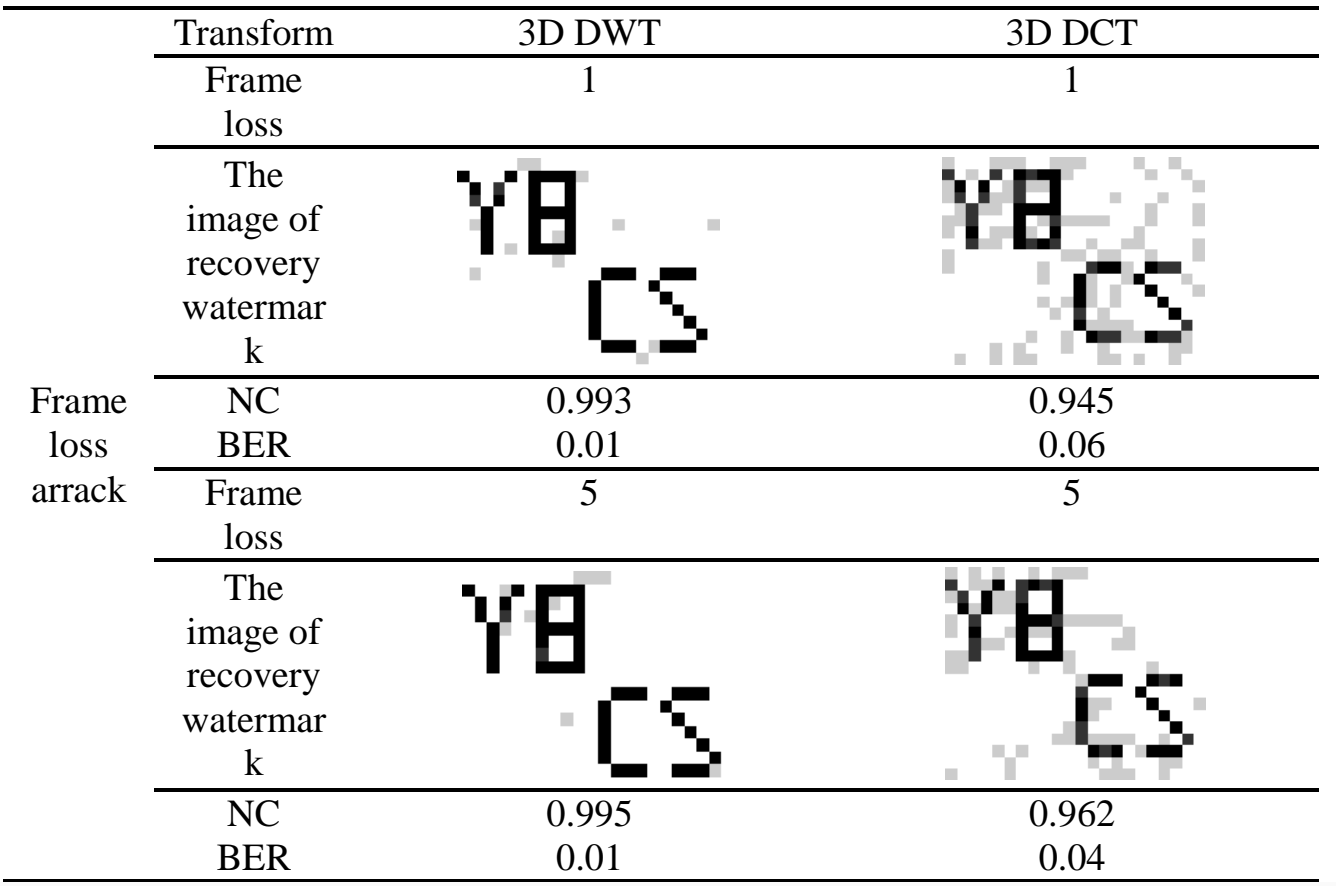

As can be seen from the Table 5, when deleting the first frame of located in different group in some extent will have effect on the extraction results but it still can restore a recognizable watermark image. Select delete on the late location of the video frame group does not have any effect for watermark extraction.

Through the atack experimental results can be concluded that two kinds of video watermarking algorithm against noise attack and frame exchange and frame loss has better robustness. In order to make the results more clear make the experimental data by the statistical map out the following graph, as shown in Figure 8 and Figure 9.

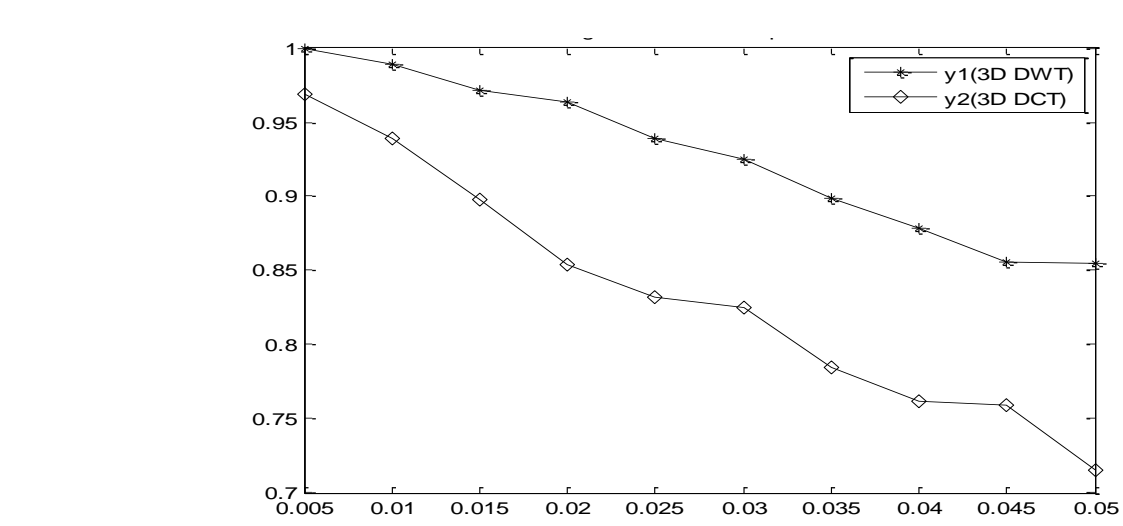

(a) The NC contrast figure of Gaussian noise attacked 


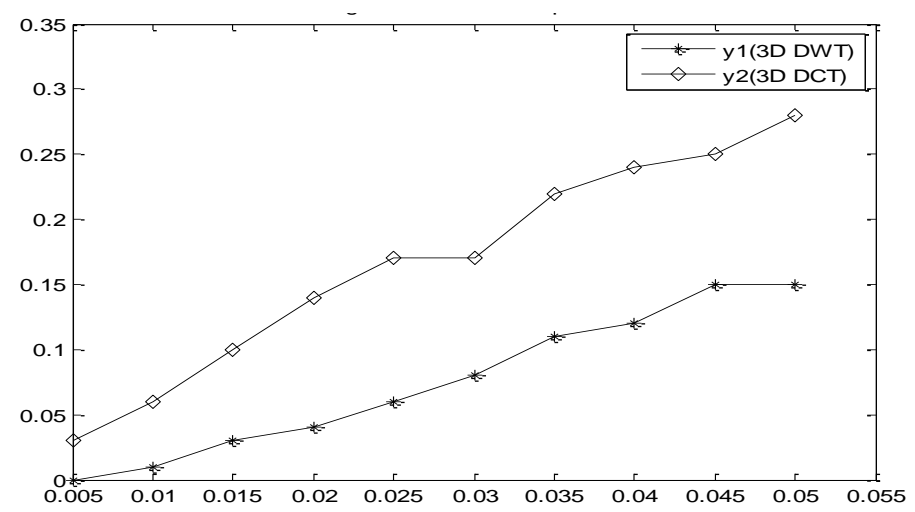

(b) The BER contrast figure of Gaussian noise attacked

Figure 8. The Contrast Figure of GaussianYNoise Attacked

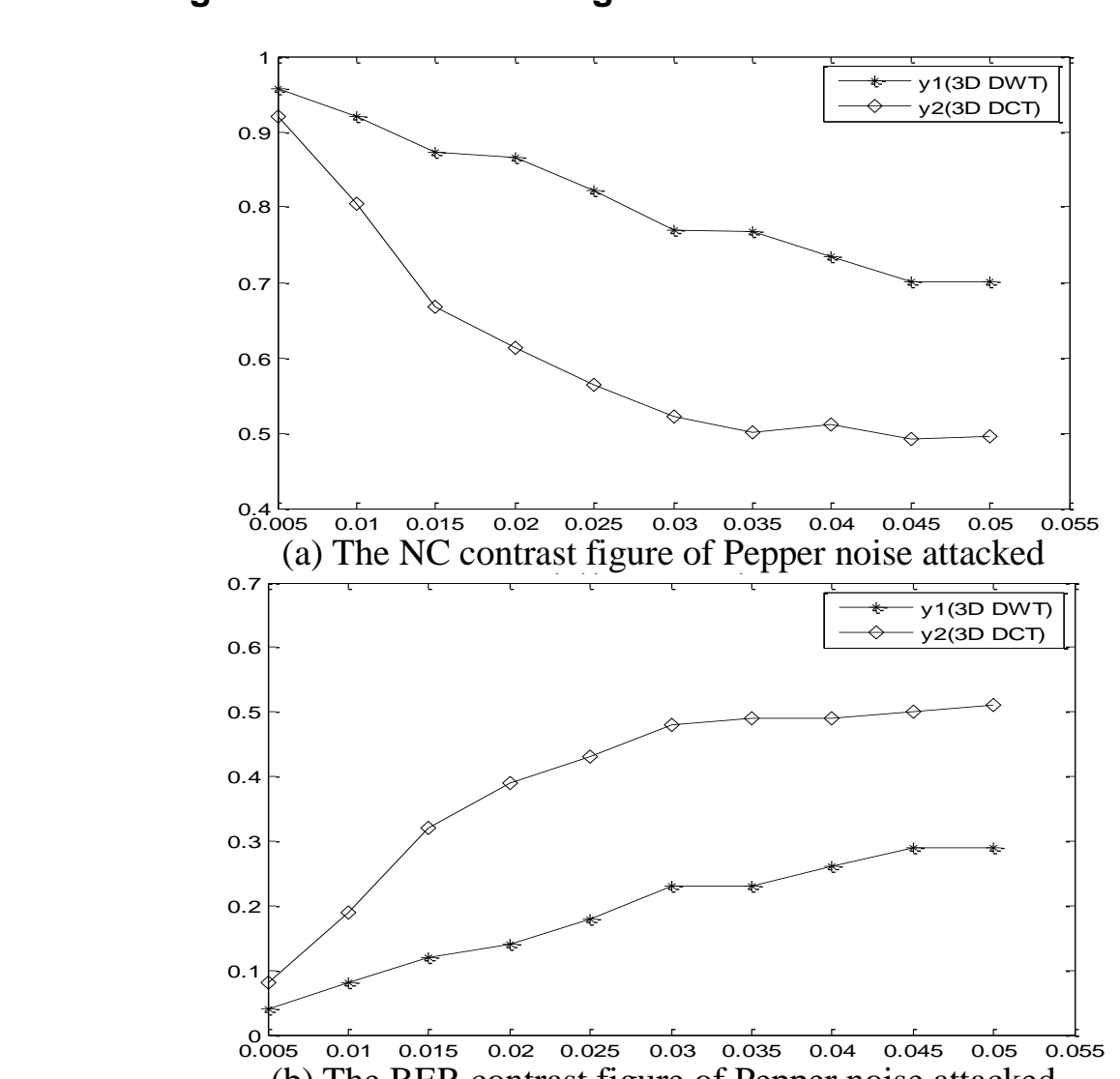

(b) The BER contrast figure of Pepper noise attacked

Figure 9. The Contrast Figure of Pepper Noise Attacked

From Figure 8 and Figure 9 we can see the video watermarking algorithm based on threedimensional discrete wavelet transform attack resistance obvious higher than that the video watermarking algorithm based on three-dimensional discrete cosine transform. Here we neglected the experiment error due to various reasons, as far as possible combined with the ideal experimental environment get the experimental data. The invisibility (PSNR) of similar cases and under the noise attacks and frame attack three dimensional discrete wavelet transform is slightly stronger robustness to reflect the discrete wavelet transform various 
advantages. But we can't say three-dimensional discrete cosine transform useless, this algorithm complexity is lower than discrete wavelet transform and it's a good robust digital video watermarking algorithm that combination of discrete cosine transform [15] and human visual system (HVS).

\section{Conclusion}

This thesis mentioned three-dimensional discrete wavelet transform and threedimensional discrete cosine transform based on mean quantization algorithm of two kinds of robust digital video watermarking algorithm, for the original video proceed three dimensional transformation and embedded watermark information into the approximation part of the important coefficient that by corresponding transform to original video so that the generated watermark has good robustness and inxistbility. The attack experiment results show that the two kinds of watermarking atgorithm can resist various attacks and ensure the robustness of the watermark meantime the embedded watermark to affect the quality of the original video are also small and the human eye almost couldn't feel the video quality after embedded watermark, but compared with the experimental results of three-dimensional discrete wavelet transform on the robustness is better than that of the three-dimensional discrete cosine transform. The main reason has the following points: (1) The discrete cosine transform sometimes produce square effect. In the process of three-dimensienal discrete wavelet transform, first carried on the block processing to extract frames and in some extent the produce square affect the transformation of results. (2) The discrete wavelet transform has good space directional selectivity and agrees well with the human visual system but the discrete cosine transform does not have such directional selectivity [16]. (3) The discrete wavelet transform has the stronger ability of muti-resolution analysis. Thus, when we design of algorithm should avoid the flaw of algorithm itself at the same time need to help with the advantages of the algorithm to improve the overall performance of the algorithm. Through the research we can see, in the next period of time the digital video watermarking will become an important research topic and along with the progress of network technology and hultimedia digital technology as well as the continuous development of all kinds of network application technology, the digital video watermarking technology will inevitably encountered new problems which requires we put forward a new theory and new technology to solve new problems.

\section{Acknowledgements}

This research project was supported by the National Natural Science Foundation of China (Grant No. 61262090)

\section{References}

[1] X. Zou, C. Peng, J. T. Li and S. C. Liu, "Video watermarking technology research", Journal of engineering graphics, vol. 3, (2002), pp. 104-108.

[2] B. I. Wang, Q. Chen and F. S. Deng, "Digital watermarking technology”, Xi'an: xi'an university of electronic science and technology press, $(\mathbf{2 0 0 3})$.

[3] Z. W. Hang and Z. H. Zhang, "Video watermarking technology research", Computer engineering and science, vol. 26, no. 9, (2004), pp. 65-68.

[4] Y. Lu, Q. Y. He, R. Ding, R. H. Dai and G. H. Er, "A video watermarking algorithm based on threedimension discrete cosine transform research", The eighth national symposium on cable TV technology, (2005), pp. 44-49. 
[5] L. P. Hou and L. Shi, "Mean quantization in the audio digital watermark", The application of science and technology information, no. 33, (2009), pp. 22-24.

[6] X. G. Cheng and J. H. Zhang, "DCT watermarking algorithm and DWT watermarking algorithm performance comparison", Xiangfan college journal, vol. 28, no. 2, (2007) February.

[7] K. X. Yi, J. Y. Shi and X. Sun, "Digital watermarking technology research progress", Journal of image and graphics, (2001), vol. 6, A - 2.

[8] J. Q. Xie and C. H. Yang, "Technology research in the digital video watermarking", Journal of Hunan College of finance and economics school, (2006), vol. 22, (102).

[9] L. Lv, "Digital video watermarking technology", Journal of VIP information, (2004), vol. 64.

[10] D. W. Xu and R. D. Wang, "Based on block three-dimension wavelet transform of video watermarking algorithm. Journal of southeast university (natural science edition)", (2007), vol. 37 (supplement (I).

[11] F. Deguillarme, G. Csurka and J. O. Ruanaidh, "Robust 3D DFT video watermarking [A]", Prøceedings of SPIE Security and watermarking of multimedia Contents, San Jose, (1999), pp. 113-124.

[12] L. Liu, "The Matlab implementation of three dimensional discrete wavelet transforms Matlab", The public science and technology, vol. 5,(2006), [A] O241.86.

[13] H. S. Tan, H. M. Zhao, X. Y. Ding and Y. H. Xiao, "Three-dimension inverse discrete wavelet transform data hiding algorithm of video image", Journal of circuits and systems, yol. 16, no 3, (2011) June.

[14] P. W. Chan, M. R. Lyu and R. T. Chin, "A Novel Scheme for Hybrid Digit V/deo Watermarking: Approach, Evaluation and Experimentation [J]", IEEE Transactions on circuits and systems for video technology, vol. 15, no. 12, (2005).

[15] C. Peng and T. F. Jiang, "An image watermarking algorithm based on DCy", Information and security, (2007).

[16] Q. Y. He, "Based on the three dimensional discrete/cosine transform video watermarking research", Tsinghua University, (2005).

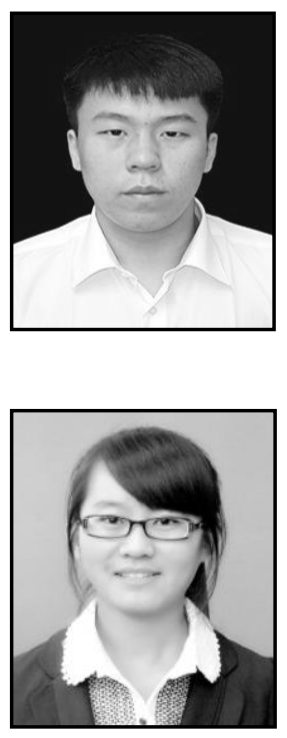

Xiao Lu, he is a postgraduate, major in Information Security, now studying at Yanbian University in China. He research interests are in the areas of copyright, protection technology, information security, digital

wåtermarking and digital forensic marking.

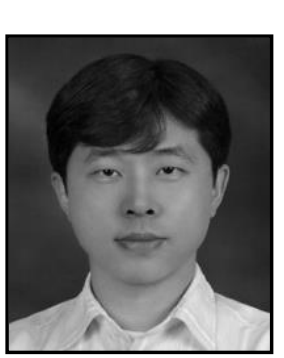

De Li, he received the Ph.D. degree from Sangmyung University, major in computer science in 2005 . He is currently a professor of Dept. of Computer Science at Yanbian University in China. He is also a Principal Researcher at Copyright Protection Research Institute, Sangmyung University. His research interests are in the areas of copyright protection technology, digital watermarking, and digital forensic marking. 
International Journal of Multimedia and Ubiquitous Engineering Vol.9, No.7 (2014)

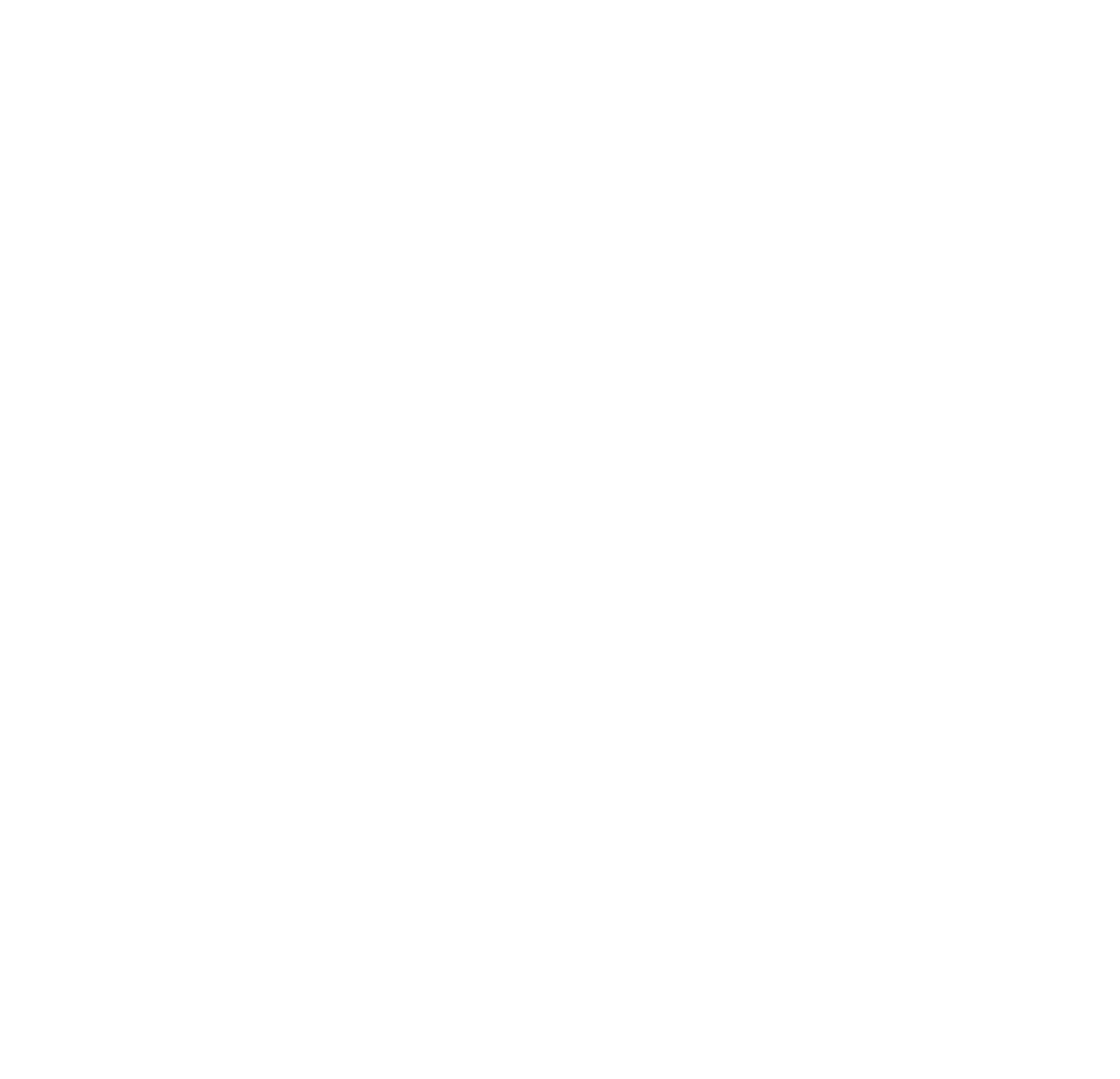

